\title{
Establishing gene regulatory networks from Parkinson's disease risk loci
}

Gene regulatory networks in $P D$

Sophie L. Farrow ${ }^{1}$, William Schierding ${ }^{1,2}$, Sreemol Gokuladhas ${ }^{1}$, Evgeniia Golovina ${ }^{1}$, Tayaza

Fadason $^{1,2}$, Antony A. Cooper ${ }^{3,4^{*}}$, Justin M. O’Sullivan ${ }^{1,2,5,6^{*}}$

1 Liggins Institute, The University of Auckland, Auckland, New Zealand

2 The Maurice Wilkins Centre, The University of Auckland, Auckland, New Zealand

3 Australian Parkinson's Mission, Garvan Institute of Medical Research, Sydney, New South Wales, Australia

4 St Vincent's Clinical School, UNSW Sydney, Sydney, New South Wales, Australia

5 Brain Research New Zealand, The University of Auckland, Auckland, New Zealand

6 MRC Lifecourse Epidemiology Unit, University of Southampton, United Kingdom

*corresponding co-senior authors. 


\begin{abstract}
The latest meta-analysis of genome wide association studies (GWAS) identified 90 independent single nucleotide polymorphisms (SNPs) across 78 genomic regions associated with Parkinson's disease (PD), yet the mechanisms by which these variants influence the development of the disease remains largely elusive. To establish the functional gene regulatory networks associated with PD-SNPs, we utilised an approach combining spatial (chromosomal conformation capture) and functional (expression quantitative trait loci; eQTL) data. We identified 518 genes subject to regulation by 76 PD-SNPs across 49 tissues, that encompass 36 peripheral and 13 CNS tissues. Notably, one third of these genes were regulated via trans- acting mechanisms (distal; risk locus-gene separated by $>1 \mathrm{Mb}$, or on different chromosomes). Of particular interest is the identification of a novel trans-eQTLgene connection between rs10847864 and SYNJ1 in the adult brain cortex, highlighting a convergence between familial studies and PD GWAS loci for SYNJ1 (PARK20) for the first time. Furthermore, we identified 16 neuro-development specific eQTL-gene regulatory connections within the foetal cortex, consistent with hypotheses suggesting a neurodevelopmental involvement in the pathogenesis of PD. Through utilising Louvain clustering we extracted nine significant and highly intra-connected clusters within the entire gene regulatory network. The nine clusters are enriched for specific biological processes and pathways, some of which have not previously been associated with PD. Together, our results not only contribute to an overall understanding of the mechanisms and impact of specific combinations of PD-SNPs, but also highlight the potential impact gene regulatory networks may have when elucidating aetiological subtypes of PD.
\end{abstract}




\section{Introduction}

Parkinson's disease (PD) is considered to be primarily an idiopathic neurodegenerative disorder, with monogenic forms contributing to just $5-10 \%$ of all cases ${ }^{1}$. However, the idiopathic nature of PD is being questioned, as evidence increasingly supports a complex involvement of genetics in the development of the majority of cases ${ }^{2,3}$. Genome-wide

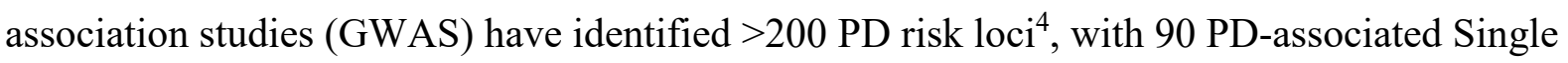
Nucleotide Polymorphisms (PD-SNPs) across 78 risk loci reported in the largest metaanalysis to date ${ }^{5}$. As is typically observed with GWAS variants, the majority of the PD-SNPs are located within non-coding regions of the genome, with no direct or obvious influence on protein structure or function ${ }^{6,7}$. Studies have shown that such non-coding disease-associated variants are more likely to be located within regulatory regions ${ }^{8}$, and thus contribute to risk through influencing gene regulation and expression, either locally or distally. These regulatory interactions are likely to be tissue specific, adding a further layer of complexity. Consequently, determining how these variants contribute to PD risk, both individually and in combination, poses a major scientific challenge ${ }^{9,10}$.

Although PD is defined as a neurodegenerative disease, mounting evidence demonstrates the role of non-central nervous system (CNS) tissues in the development and presentation of such disorders (i.e. Huntington's ${ }^{11}$ and $\mathrm{PD}^{12,13}$ ). Both alpha-synuclein ( $\left.\alpha \mathrm{Syn}\right)$ protein pathology and modulation of PD-related genes have been identified in peripheral tissues (e.g. the gastrointestinal tract and heart) of PD patients ${ }^{12,14-19}$. The contribution of peripheral tissue in the origins of PD warrants further research, and thus the consideration of how PD-SNPs mediate risk should not be confined to tissues of the CNS.

Spatial gene regulatory interactions are hypothesized to be drivers of complex trait heritability ${ }^{20}$, acting through both cis- (nearby) and trans- (distal; locus-gene separated by > 
$1 \mathrm{Mb}$, or on different chromosomes) mechanisms (Figure 1) $)^{19,21,22}$. These cis- and trans-acting elements can regulate the transcription of one or more genes, in a tissue specific manner, and are commonly detected in the form of expression quantitative trait loci (eQTL) ${ }^{23}$. Genetic variation within elements of gene regulatory networks likely confer risk at different developmental stages, including during foetal neurodevelopment - a critical stage that has a growing body of support in neurodegenerative diseases ${ }^{24}$.

Here we performed correlational analyses of experimentally derived data to identify eQTLs that physically connect PD-SNPs to the genes that they control, in three-dimensions, with the goal of understanding the putative functional impacts of known PD-SNPs ${ }^{21}$. The integration of spatial and eQTL data allows for the identification of trans-eQTL-gene associations ${ }^{25}$, thereby nominating genes which have not previously been implicated in PD. Our analysis identified 518 genes subject to regulation by 76 PD-SNPs across 49 tissues. Further, clustering analysis of the entire gene network revealed nine significant, intra-connected clusters, enriched for both novel and known PD biological pathways, highlighting putative disease-causative molecular mechanisms and areas for future research. 


\section{Methods}

\section{Data and reference files}

The 90 PD-SNPs (across 78 genomic regions; Supplementary table 1) investigated in this study were previously identified by a GWAS meta-analysis as being of genome wide significance $\left(\mathrm{p}<5 \times 10^{-8}\right)^{5}$. The human genome reference sequence used in this study was GRCh38 (hg38).

\section{Identification of eQTL-gene pairs}

The contextualise developmental SNPs using 3D information (CoDeS3D) ${ }^{21}$ algorithm was used to identify genes whose transcript levels are putatively regulated by the 90 PD-SNPs. CoDeS3D integrates data on spatial interactions between genomic loci (Hi-C data; Supplementary table 2) with expression data (genotype-tissue expression database version 8 ; GTEx $v 8^{26}$ ) to identify genes whose transcript levels are associated with a physical connection to the SNP (i.e. spatial eQTL).

Hi-C captures regions of the genome that are physically interacting, and can be covalently connected by a cross-linking agent ${ }^{27}$. The hg38 reference genome was digitally digested with MboI, DpnII and HindIII to obtain all possible Hi-C fragment locations for the 90 PD-SNP loci. All identified SNP fragments (tagged by the PD-SNPs) were then queried against the Hi-C databases (70 different cell lines from 12 studies; Supplementary table 2) to identify distal fragments of DNA that spatially connect to the SNP loci. Spatial SNP-gene connections are established when the SNP-containing fragment spatially connects to a fragment that overlaps any region between the start and end of a gene as defined by GENCODE. There was no binning or padding around restriction fragments to obtain gene overlap. The resulting spatial SNP-gene pairs were subsequently used to query the GTEx v8 eQTL database ${ }^{26}$ to 
identify spatial SNP-gene pairs with significant eQTLs (both cis- and trans- acting eQTL; false discovery rate (FDR) adjusted $p<0.05)$.

We performed a 'brain specific' analysis by interrogating only the subset of Hi-C libraries derived from brain-specific cell lines (11 cell lines from 4 studies, highlighted in red in Supplementary table 2$)^{28-31}$ and only expression data from the 13 brain-specific tissues in GTEx v8.

\section{Identification of neurodevelopmental-specific eQTL-gene pairs}

We performed a neurodevelopmental stage specific analysis by interrogating Hi-C libraries from foetal-specific brain cell lines (cortical plate neurons; germinal zone neurons; Supplementary table 2; datasets no. 1 and 2) with expression data from a foetal cortex eQTL dataset $^{32}$.

\section{Functional analysis of eQTL SNPs}

SNPnexus v4 ${ }^{33}$ (https://www.snp-nexus.org/v4/; accessed 22/06/2020) was used to obtain known epigenomic annotations for the eQTL SNPs.

\section{Probability of gene loss of function intolerance}

The LOEUF (loss-of-function observed/expected upper bound fraction) ${ }^{34}$ score for the genes, within the significant SNP-gene pairs, was obtained from gnomAD v2.1.1 $1^{35}$ (https://gnomad.broadinstitute.org/; accessed 21/07/2020) to determine the level of constraint on the identified genes.

\section{Protein-protein interaction (PPI) and Modularity clustering}

$\mathrm{STRING}^{36}$ (Search Tool for Retrieval of Interaction Genes/proteins; https://string-db.org, accessed 22/07/2020) was queried to identify published information on interactions between genes, or their respective proteins. Only PPIs with a high confidence level $(>0.700$, as defined in STRING) were used for this analysis. A Louvain method was used to determine the syntality of each node, following four different criteria: 1) immediate connection; 2) 
shortest path (i.e. the minimum number of edges connecting any two nodes); 3) node acting as a bridge; 4) connections that nodes have in common. The proteins were then hierarchically clustered using the Louvain algorithm ${ }^{37}$, clusters were defined as significant if $\mathrm{p}<0.05$.

\section{Pathway analyses \& literature search}

The g:Profiler ${ }^{38}$ database was used to identify enriched pathways. Queries were run on: 1) all genes' 2) the 'cis'; and 3) the 'trans' subsets of genes (i.e. genes regulated only in cis, or only in trans). PubMed was used to search (using “[gene name]" and Parkinson's") the published literature for all identified genes and known PD associations.

\section{Additional analyses of genes and variants identified by Makarious et al}

Makarious et al recently utilised a multi-modality approach to identify genetic and transcriptomic features that contribute to risk predictions of $\mathrm{PD}^{39}$. They highlighted two SNPs (rs10835060 and rs4238361), and 29 genes. We performed CoDeS3D analysis on the two SNPs, across all Hi-C cell lines and GTEx tissues (as previously described). The resulting eQTL gene pairs, along with the 29 genes highlighted through transcriptomic analysis, were combined with our set of 523 genes. Louvain clustering and PPI analysis were re-run on this combined list of genes to see how or if the subset of genes co-locate within the networks.

\section{URLs}

CoDeS3D pipeline: https://github.com/Genome3d/codes3d-v2

gnomAD: https:/gnomad.broadinstitute.org/

gProfiler: https://biit.cs.ut.ee/gprofiler/

UCSC: https://genome.ucsc.edu/index.html

STRING: https://string-db.org/

SNPNexus: https://www.snp-nexus.org/

Louvain clustering analysis: https://github.com/Genome3d/PPI-network-analysis

\section{Data Availability}


All data generated during this study are included in the supplementary information. Datasets analysed and tools used in this study were all derived from publicly available resources (See URLs). 


\section{Results}

\section{PD GWAS SNPs regulate the expression of $>500$ genes}

Nalls et al. ${ }^{5}$ identified 90 SNPs that were associated with PD at the level of genome-wide significance (Supplementary table 1), yet the mechanisms by which these variants influence the development of the disease remains largely elusive. We used the CoDeS3D algorithm ${ }^{21}$ to identify SNPs that have evidence of physical interaction with the gene as captured by Hi-C (Supplementary table 2) and also associate with changes in gene expression (hereafter eQTLs) and the genes whose transcript levels were affected (Figure 1).

76 (84\%) of the 90 PD risk SNPs were identified as eQTLs associated with the regulation of 518 genes through 542 unique eQTL-gene pairs, across the 49 tissues (Table 1, Supplementary table 3). The 76 eQTLs were individually associated with the regulatory impacts of as few as one, or as many as 39 genes in cis and trans (Supplementary figure 1). We identified 178 of the 542 genes as being associated with PD through trans-eQTL-gene connections. We did not identify eQTL interactions for 14 of the 90 SNPs. Conversely, 8 of these 14 SNPs are annotated as being eQTLs in the IPDGC GWAS Locus Browser ${ }^{40}$, and a further four are eQTLs in GTEx (Supplementary table 1). However, in these 12 instances, the eQTLs occur in cis- (i.e. within $1 \mathrm{Mb}$ ) and are not supported by Hi-C data.

\begin{tabular}{|lll|} 
& \multicolumn{2}{l|}{ PD SNPs } \\
\cline { 2 - 3 } & Brain specific* & All tissues* \\
\hline No. SNPs & 90 & 90 \\
No. eQTL SNPs $^{\#}$ & 55 & 76 \\
No. Genes $^{\uparrow}$ & 165 & 518 \\
No. eQTL-Gene pairs $^{*}$ & 167 & 542 \\
No. trans eQTL-Gene pairs & 30 & 178 \\
\hline
\end{tabular}

Table 1: Summary statistics for the spatial eQTL-gene regulatory network for the $90 P D$ -

SNPs. SNPs were downloaded from Nalls et al 2019 GWAS paper (download date:

18.06.2020). ${ }^{*} Q T L S N P$ s were defined as having significant spatial interactions (FDR 
$\leq 0.05)$ with at least one gene. "genes were those whose expression was shown to be affected by an eQTL SNP. ${ }^{¥}$ The total number of SNP-gene pairs reflects interactions with FDR $\leq 0.05$ in at least one GTEx tissue. * For full list of tissues see Supplementary tables 3 and 4.

Consistent with observations for SNPs associated with other traits ${ }^{41}$, at least one transregulatory interaction was identified for $81.6 \%$ (62 of 76) of the eQTLs. Moreover, $92.7 \%$ (165 of 178) of these trans-eQTL-gene interactions were identified in only one tissue. By contrast, the cis- interactions were identified in eight tissues on average (range of 1 to 49 tissues). $11.8 \%$ of the eQTLs (9 of 76; Supplementary table 1) were exclusively involved in trans-regulatory interactions. Trans-eQTL interactions regulated $18.1 \%$ of the genes identified in the brain ( 30 out of 166 ; Supplementary table 4 ), and $32.8 \%$ of the genes amongst all 49 tissues (178 out of 518; Table 2; Supplementary table 3). Collectively, these results highlight the importance of looking beyond the nearest gene to identify the regulatory effects of disease-associated variants.

\begin{tabular}{|lll|}
\hline & \multicolumn{2}{l}{ Genes subject to cis- or trans- regulation } \\
\cline { 2 - 3 } & Brain specific* & All tissues* \\
\hline $\begin{array}{lll}\text { Cis eQTL-gene pairs } \\
\text { Trans-intrachromosomal }\end{array}$ & $136(82.0 \%)$ & $364(67.2 \%)$ \\
eQTL-gene pairs & $10(6.0 \%)$ & $56(10.3 \%)$ \\
$\begin{array}{l}\text { Trans-interchromosomal } \\
\text { eQTL-gene pairs }\end{array}$ & $20(12.0 \%)$ & $122(22.5 \%)$ \\
\hline
\end{tabular}

Table 2: Proportion of genes subject to cis- and trans- regulation. The proportion of eQTLgene pairs that are either cis-, trans-intrachromosomal or trans-interchromosomal in (a) 13 GTEx brain-specific tissues and (b) all 49 GTEx tissues. Brain-specific indicates the eQTL dataset obtained through analysing $\mathrm{Hi}$-C cell lines only from the brain, and eQTLs only from the brain tissues in GTEx. All-tissues indicates the eQTL dataset obtained through analysing all Hi-C cell lines, and eQTLs from all tissues in GTEx. There is a significant difference (Chi 
square test p.value <0.01) between brain tissues and all tissues for the proportions of the cis vs. trans eQTLs.

We reasoned that SNPs that are involved in eQTLs likely mark enhancer or promoter sites ${ }^{42}$. We queried SNPnexus ${ }^{33}$ to identify those eQTLs that were marked by histone modifications or fell within open chromatin regions, as indicated by DNAse accessibility. Consistent with our hypothesis, $91 \%$ (69 of 76) of the SNPs were marked by histone modifications associated with either enhancers (58) and/or promoters (27). 27.6\% of the SNPs were within accessible chromatin(Supplementary table 5). Collectively, these results are consistent with the hypothesis that the loci marked by these eQTLs may be involved in the regulation of gene expression.

Pathway analysis was conducted on the complete set of 518 genes that were impacted by the eQTLs (Supplementary table 6). g:Profiler ${ }^{38}$ identified significant (adj.p < 0.05) enrichment within 10 known biological pathways (g:GOSt), including response to interferon-gamma, synaptic vesicle recycling and endocytosis.

\section{The regulatory impact of PD-SNPs extends beyond the CNS}

Although PD is considered a degenerative disease of the brain, it has become apparent that dysfunction and or alpha-synuclein pathology is observed in non-CNS tissues of PD patients $^{13-15}$. Our spatial eQTL analysis included an assessment of the tissue distribution of the effects of the identified eQTLs within 13 CNS and 36 peripheral tissues. We identified peripheral tissue-specific eQTLs for $28 \%$ of the PD-SNPs (21 of 76). Only 2 of the 76 PDSNPs (i.e. rs10756907 - SH3GL2, brain cortex; rs873786 - SLC26A1, brain cerebellum) had eQTLs that impacted gene expression levels exclusively in the brain. This supports a possible role for peripheral tissues in PD risk (Supplementary figure 3). 
The ability to detect eQTLs in specific tissues is known to correlate with tissue sample size within $\mathrm{GTEx}^{43}$. Consistent with this, we identified highly significant correlations between tissue sample numbers and a) all-eQTLs in the brain tissues (Figure 2a; identified using brain specific Hi-C and eQTL data; Supplementary Table 4); and b) all tissues (i.e. the 49 tissues included within GTEx; Figure 2e). These highly significant correlations remained when analysing the $c i s$-eQTL subsets in the brain $\left(\mathrm{R}=0.93, \mathrm{p}=3.6 \mathrm{e}^{-06}\right.$; Figure $\left.2 \mathrm{~b}\right)$, and all tissues $\left(\mathrm{R}=0.9, \mathrm{p}<2.2 \mathrm{e}^{-16}\right.$; Figure $\left.2 \mathrm{f}\right)$. Similarly, the correlation was evident for transintrachromosomal eQTLs detected in all tissues $\left(\mathrm{R}=0.67, \mathrm{p}<1.8 \mathrm{e}^{-06}\right.$; Figure $\left.2 \mathrm{~g}\right)$. By contrast, there was no observable correlation between the number of trans-interchromosomal interactions and tissue sample number (Figure 2d,h). The substantia nigra and brain cerebellar hemisphere exhibited more trans-interchromosomal-eQTLs (Figure 2d), while the thyroid exhibited more eQTLs than expected across all three categories (Figure 2e-h).

\section{Genes subject to trans-regulation by PD-SNPs are more likely to be intolerant to loss-of-function mutations}

Genes that are intolerant to inactivation by loss-of-function variants are deemed essential for healthy development ${ }^{44}$. Intolerance to loss of function variants leaves changes to regulation as one of the few mechanisms that can be modified to introduce variation at a population level. The 117 trans-interchromosomal-eQTL regulated genes were significantly $(\mathrm{p}<0.01$, Kruskal-Wallis test) more intolerant to loss-of-function mutations (LOEUF 0.42 [median]; a low LOEUF score is indicative of evolutionary constraint) than those regulated by cis- or trans-intrachromosomal acting eQTLs (LOEUF 0.83 and 0.85 respectively [median]; Figure 3; Supplementary table 7). This result is consistent with earlier observations that trans-eQTLs are enriched in regulating constrained genes with low LOEUF scores ${ }^{19}$. 


\section{PD GWAS SNPs regulate expression of a subset of genes within the foetal cortex}

Emerging evidence suggests PD has a neuro-developmental aspect ${ }^{48}$, similar to recent observations in Huntington's disease ${ }^{24}$. Therefore, we analysed the regulatory impacts of the PD-SNPs using foetal cell line Hi-C (i.e. cortical plate neurons and germinal zone neurons ${ }^{29}$ ) and foetal cortex eQTL datasets ${ }^{32}$ (Supplementary table 8). 33 genes were found to be regulated by 22 PD-SNPs in the foetal cortex. Of these, sixteen genes were regulated by eQTLs involving PD-SNPs in the foetal cortex, without evidence of any eQTLs in adult brain tissues (Figure 4; Supplementary table 4). Ten genes were affected by eQTLs involving PDSNPs in both the foetal and adult cortex, with effect sizes that were similar in both (Figure 4). Finally, seven genes were regulated by cis-eQTLs in the foetal cortex and adult non-cortical brain tissues (Figure 4). These findings are consistent with the hypothesis that developmentstage specific eQTL patterns impact on disease-relevant mechanisms and thus may contribute to the proposed temporal phases of PD pathogenesis ${ }^{47}$.

\section{Louvain clustering highlights nine intra-connected protein clusters, enriched for disease-relevant, biological pathways}

Network representations of complex datasets can aid the identification of biological relationships that are often not identified by enrichment analyses ${ }^{48}$. We used a Louvain clustering algorithm to identify clusters of interacting genes and proteins from within a Protein-Protein Interaction Network (PPIN) generated from the 523 eQTL regulated genes (518 adult tissue eQTLs and the 5 unique foetal cortex eQTLs; Supplementary table 9). Nine significant $(\mathrm{p}<0.05)$ clusters consisting of 122 genes were identified within the highconfidence PPIN (Figure 5). The genes within each cluster were regulated by between 5 and 18 PD-SNPs (Supplementary table 9) and every cluster contained at least two genes that were 
co-regulated by a single SNP (Supplementary figure 4). Notably, genes that were subject to trans-acting eQTLs were central to the definition and identification of several clusters (Figure 5). Pathway analysis (g:Profiler) of the genes within the individual clusters revealed enrichment in categories that included immunological surveillance (cluster 7), synaptic vesicle recycling (cluster 5) and microtubule polymerisation (cluster 3) (Supplementary table $10)$.

Makarious et al recently used a multi-modal machine learning approach, incorporating multiomics datasets, to inform and improve predictions of $\mathrm{PD}^{39}$. Beyond the 90 GWAS SNP signals (which collectively were the top genetic feature), they also identified rs10835060 and rs4238361 as two SNPs that impact on PD biology. CoDeS3D analysis identified eQTLs for both rs10835060 (KRTAP5-AS1, CCDC88A, KRTAP5-5, BRSK2) and rs4238361 (USP47; and RP11-507B12.2 and RP11-259A24.1) (Supplementary table 11). Notably, BRSK2 colocates with cluster 2 through an established interaction with the Tau encoding MAPT gene ${ }^{49}$. The model also highlighted 29 genes through transcriptomic analysis. Three of these genes (MMP9, TRIM4 and SYS1) integrate into clusters 1, 4 and 5, respectively (Supplementary figure 5). The co-location of genes and eQTLs, identified as being important for PD $\operatorname{diagnosis}^{39}$, within the nine clusters supports the potential importance of the gene-gene interactions and enriched pathways in PD.

402 genes did not segregate in the nine clusters. Of note, 211 of the 402 genes $(52.5 \%)$ had not previously been associated with PD GWAS loci (iPDGC PD browser ${ }^{40}$ ). Of the 211,123 genes were regulated by trans-eQTL-gene connections. Notably, iPDGC identified five genes (DNAI1, EYA4, LYVE1, MYO5B, PDZRN4) as being regulated by cis-eQTLs yet. These five genes were exclusively identified through trans-eQTL regulatory connections in our analysis. 


\section{Discussion}

Assigning functionality to PD-SNPs is a critical step towards determining how they contribute to the risk of PD development. In this study, we identified 518 genes whose expression was regulated in cis or trans by PD-SNPs, and the tissues where this regulation occurs. We also demonstrated that 22 PD-SNPs impact the regulation of a subset of 16 genes solely in the foetal cortex, and a further 10 genes in both the foetal and adult cortex. Of all 523 identified genes, a subset of 122 cis- and trans- regulated genes formed nine clusters within a protein:protein interaction network that were enriched for specific biological pathways, some of which have not been previously associated with PD. Our findings support the hypothesis that both cis- and trans- dysregulation of gene expression contributes to the risk of PD and provide insight into possible disease-causing mechanisms.

SYNJ1 encodes synaptojanin-1, a presynaptic phosphoinositide phosphatase that dephosphorylates $\mathrm{PI}(4,5) \mathrm{P}_{2}$ to trigger the removal of the clathrin coat during synaptic vesicle recycling ${ }^{50} . S Y N J 1$ is a highly constrained gene $($ LOEUF score $=0.33)$ and rare missense mutations in SYNJ1 have been linked to early-onset Parkinsonism ${ }^{51}$. Despite this, GWA studies have not identified any SNPs proximal to SYNJ1 as being significantly associated with PD, nor have they attributed any significant PD SNP (near or far) to SYNJ1. Critically, we identified that the PD-associated SNP rs10847864 acts as a trans-acting eQTL for SYNJ1 expression. rs 10847864 is intronic to, and also acts as a cis-eQTL with, HIP1R, another gene that is involved in clathrin mediated endocytosis ${ }^{52-54}$. Our discovery of the trans-eQTLSYNJ1 connection merges observations from population level (i.e. GWAS) and familial studies, reinforcing the potential importance of SYNJ1 in PD. 
Our analysis identified trans-eQTLs for approximately two-thirds of the known PD-SNPs. Of note, $R A I 14$ (retinoic acid induced 14) is regulated by two trans-eQTLs, involving two independent PD-SNPs (rs2251086, chr.15 and rs55818311, chr.19). Although not yet directly linked to $\mathrm{PD}, \mathrm{RAII} 4$ (and its encoded protein ankycorbin) has been shown to play a role in the inflammatory response in glial cells ${ }^{55}$, and in the establishment of neuronal morphology ${ }^{56}$; both of which are pathways of known importance in PD pathogenesis ${ }^{57}$. Retinoic acid, a regulator of RAI14 (one of multiple roles of retinoic acid), is being explored as a potential therapeutic target for $\mathrm{PD}^{58}$.

Our results provide support for the role of peripheral tissues in PD, notably the oesophagus and thyroid. Firstly, the oesophagus is enriched for cis and trans regulatory eQTLs. rs76904798 $(\mathrm{PD}$ odds ratio $(\mathrm{OR})=1.155)$ is an eQTL that upregulates $L R R K 2$ expression in 19 peripheral tissues, including in the oesophagus. Notably, this cis-eQTL with LRRK2 is not identified in any CNS tissues. Secondly, we identified the thyroid tissue as being enriched for eQTLs, many of which were not represented in CNS tissues. The thyroid is a component of the dopaminergic system and hypothalamic-pituitary-thyroid axis network ${ }^{61}$. A potential link between thyroid hormone disorders, PD risk, and symptom severity has been suggested ${ }^{62}$. Specifically, one study identified patients with hypothyroidism to have a two-fold elevated risk of developing $\mathrm{PD}^{63}$. Collectively, these findings support the growing body of evidence for the importance of the oesophagus ${ }^{64,65}$ and thyroid ${ }^{62}$ in PD.

We hypothesised that genes regulated by PD-SNPs in foetal cortical tissue may contribute to potential neurodevelopmental aspects of $\mathrm{PD}^{24,45,46}$. Sixteen genes were regulated by PD-SNP eQTLs within the foetal cortex. Two of these genes, CNTNAPI and GALC are particularly notable. CNTNAP1 encodes Caspr1, a Neurexin family membrane protein. Reductions in 
Caspr 1 concentrations delay cortical neuron and astrocyte formation in the mouse developing cerebral cortex ${ }^{66}$. GALC encodes a lysosomal galactosylceramidase that ensures normal turnover of myelin ${ }^{67}$ and has been linked to neuronal vulnerability ${ }^{68}$. While connections between the remaining 14 genes and PD development are less clear, we speculate that SNP mediated regulation of these genes specific to the foetal cortex may contribute to early neurodevelopmental disturbances that render an individual more susceptible to PD.

Our analyses identified nine clusters that are enriched for specific biological processes and pathways, some of which have not previously been associated with PD. Dysregulated expression of the components within these pathways is potentially the basis of the risk conferred by the PD-SNPs. The clusters aid in understanding how PD SNPs mechanistically contribute to disease risk, and some interesting points can be drawn from these. For example, genes within cluster 6 are enriched for functions in DNA replication and repair, a pathway previously associated with the development of other neurodegenerative diseases ${ }^{69}$. Notably, $B R C A 1$ and RPA2 (both previously linked to DNA damage response and repair $^{70,71}$ ) are regulated in trans by PD-SNPs (rs11950533; rs9568188; rs62053943) and are central to cluster 6 . It is notable that cluster 6 contains several factors associated with PARP1 activity (e.g. the PARP1 binding protein (PARPBP) and BRCA1) that link this cluster to the repair of SSBs which are enriched at neuronal enhancers in post-mitotic neurons ${ }^{72}$. A further example is the regulation of autophagy initiation by PD-SNPs, highlighted in cluster 8 . Three interacting proteins within cluster 8, encoded by $V M P 1, B E C N 1$, and $A T G 14$ are each regulated by a different PD-SNP (rs12951632 chr. 17; rs11158026 chr. 14; rs10748818 chr. 10; Supplementary table 3), including a trans-eQTL connection regulating VMP1. rs $12951632(\mathrm{OR}=0.93)$ and $\mathrm{rs} 11158026(\mathrm{OR}=0.919)$ are protective PD-SNPs that respectively increase $B E C N 1$ and $A T G 14$ expression, two interacting core components of the 
PI3-kinase complex, required for autophagosome formation ${ }^{73}$. Individuals with both of these variants would potentially have increased autophagic capacity relative to individuals with one variant.

The genes in cluster 7 are strongly enriched for antigen processing and presentation - which is increasingly being implicated in the progression of $\mathrm{PD}^{74}$. Both rs504594 and rs9261484 are associated with a reduced risk of developing $\mathrm{PD}(\mathrm{OR}=0.8457$ and $\mathrm{OR}=0.9385$ respectively). We identified a spatial eQTL between rs504594 and HLA-DRB1 in both the foetal cortex and adult brain (including cortex and $\mathrm{SN}$ ), implicating this regulatory eQTLgene connection in both the neurodevelopment and neurodegenerative stages of PD. Interestingly, rs504594 (previous ID: rs112485776) was recently validated in a SNP-level meta-analysis $(\mathrm{OR}=0.87)$, with results displaying no residual HLA effect in adults after adjusting for the $\mathrm{SNP}^{75}$. Instead, three amino acid polymorphisms within the $H L A-D R B 1$ gene were identified as drivers of the association between the HLA region and PD risk $^{75}$. We agree that the impacts of rs504594 are contingent upon the $H L A-D R B 1$ allele - both in terms of regulation and protein sequence. We contend that the effects of the rs504594 eQTL are developmental. Future studies must untangle these developmental effects and identify the neurodevelopmental stages that may prime certain individuals to be more vulnerable to later triggering mechanisms. We note that such interpretation should be taken with caution given the highly polymorphic nature of the HLA-region.

We acknowledge several limitations to our analysis. Firstly, the Hi-C cohort and GTEx libraries were generated from unrelated samples that were not age or gender matched. Secondly, the sampled donors in GTEx are predominantly of European descent, limiting the significance of our findings to this ethnicity. However, the GWAS cohort also used 
individuals of European ancestry, meaning for this analysis the datasets were congruent. Thirdly, our eQTL analysis assumes that mRNA concentration correlates directly with protein levels. While it is true that protein levels are to some extent determined by their mRNA concentration, there are many post-transcriptional processes that can lead to a deviation from the expected correlation ${ }^{76}$. The fourth limitation is that eQTL data represent composite datasets across developmental periods (e.g. foetal samples were aged from 14-21 weeks post-conception and the adult samples were from individuals aged 21-70 years). Despite such limitations, the identification of trans-eQTLs is a particular strength of our methodology, relying on captured contacts within the genome organisation to reduce the impact of multiple testing correction. As such, we contend that these limitations do not invalidate the significance of our findings of trans-acting eQTLs and the genes that they impact.

\section{Conclusions}

Understanding the functional impact of PD-SNPs is critical to our understanding of how these variants contribute to the development and clinical presentation of PD. Our functional interpretation of PD-associated SNPs integrates individual loci into a gene regulatory network, which includes genes with and without prior PD associations. The regulatory network includes clusters, and within them genes, that are enriched for biological functions that have known, putative or previously unknown roles in PD. Development-specific changes to this network (within the foetal cortex) are suggestive of roles for neurodevelopmental changes being early contributors to PD disease risk. Similarly, enrichments for regulatory changes within peripheral tissues may indicate a greater role for these tissues in PD than is currently appreciated. Collectively, our findings not only contribute to an overall understanding of the multiple biological pathways associated with PD risk loci, but also 
bioRxiv preprint doi: https://doi.org/10.1101/2021.04.08.439080; this version posted April 9, 2021. The copyright holder for this preprint (which was not certified by peer review) is the author/funder, who has granted bioRxiv a license to display the preprint in perpetuity. It is made available under aCC-BY-NC-ND 4.0 International license.

highlight the potential utility of gene regulatory networks when considering etiological subtypes of PD. 


\section{Acknowledgements}

SF, WS, TF, AC, and JOS were funded by the Michael J Fox Foundation for Parkinson's research and the Silverstein Foundation for Parkinson's with GBA - grant ID 16229 to JOS. SF and TF were funded by the Dines Family Charitable Trust. AC received grant funding from the Australian government. SG was funded by an MBIE Catalyst Grant (The New Zealand-Australia LifeCourse Collaboration on Genes, Environment, Nutrition and Obesity (GENO); UOAX1611). EG was funded by University of Auckland Doctoral Scholarship.

\section{Author Contributions}

SF performed analyses and wrote the manuscript. TF and EG contributed to the preparation of eQTL and Hi-C datasets used in the manuscript. TF and WS contributed to the development of CoDeS3D. SG contributed to the network analysis script and the associated dataset. TF, EG, and SG all commented on the manuscript. WS and AC advised on the study and co-wrote the manuscript. JOS led the study and co-wrote the manuscript.

\section{Competing Interests Statement}

The authors declare no competing interests

\section{Supplementary Material}

Supplementary tables are available at:

https://github.com/sfar956/PDGWAS regulatorynetworks 


\section{References}

1. Bandres-Ciga, S., Diez-Fairen, M., Kim, J. J. \& Singleton, A. B. Genetics of

Parkinson's disease: An introspection of its journey towards precision medicine.

Neurobiology of Disease 137, 104782 (2020).

2. Billingsley, K. J. et al. Mitochondria function associated genes contribute to

Parkinson's Disease risk and later age at onset. npj Park. Dis. 5, 1-9 (2019).

3. Blauwendraat, C., Nalls, M. A. \& Singleton, A. B. The genetic architecture of

Parkinson's disease. Lancet Neurol. (2020). doi:10.1016/s1474-4422(19)30287-x

4. Buniello, A. et al. The NHGRI-EBI GWAS Catalog of published genome-wide association studies, targeted arrays and summary statistics 2019. Nucleic Acids Res. 47, D1005-D1012 (2019).

5. Nalls, M. A. et al. Identification of novel risk loci, causal insights, and heritable risk for Parkinson's disease: a meta-analysis of genome-wide association studies. Lancet Neurol. 18, 1091-1102 (2019).

6. Edwards, S. L., Beesley, J., French, J. D. \& Dunning, M. Beyond GWASs:

Illuminating the dark road from association to function. American Journal of Human Genetics 93, 779-797 (2013).

7. Porcu, E. et al. Mendelian randomization integrating GWAS and eQTL data reveals genetic determinants of complex and clinical traits. Nat. Commun. 10, 1-12 (2019).

8. Maurano, M. T. et al. Systematic localization of common disease-associated variation in regulatory DNA. Science (80-. ). 337, 1190-1195 (2012).

9. Pierce, S. E. et al. Post-GWAS knowledge gap: the how, where, and when. npj Park. Dis. 6, 1-5 (2020).

10. French, J. D. \& Edwards, S. L. The Role of Noncoding Variants in Heritable Disease. Trends Genet. 0, (2020). 
11. Sassone, J., Colciago, C., Cislaghi, G., Silani, V. \& Ciammola, A. Huntington's disease: The current state of research with peripheral tissues. Experimental Neurology 219, 385-397 (2009).

12. Horsager, J. et al. Brain-first versus body-first Parkinson's disease: a multimodal imaging case-control study. Brain 143, 3077-3088 (2020).

13. Borghammer, P., Knudsen, K., Fedorova, T. D. \& Brooks, D. J. Imaging Parkinson's disease below the neck. npj Parkinson's Disease 3, 1-10 (2017).

14. Wakabayashi, K. Where and how alpha-synuclein pathology spreads in Parkinson's disease: Neuropathology 40, 415-425 (2020).

15. Gelpi, E. et al. Multiple organ involvement by alpha-synuclein pathology in Lewy body disorders. Mov. Disord. 29, 1010-1018 (2014).

16. Martinez-Valbuena, I. et al. Interaction of amyloidogenic proteins in pancreatic $\beta$ cells from subjects with synucleinopathies. Acta Neuropathol. 135, 877-886 (2018).

17. Homma, T., Mochizuki, Y. \& Mizutani, T. Phosphorylated $\alpha$-synuclein immunoreactivity in the posterior pituitary lobe. Neuropathology 32, 385-389 (2012).

18. Kim, S. et al. Transneuronal Propagation of Pathologic $\alpha$-Synuclein from the Gut to the Brain Models Parkinson's Disease. Neuron 103, 627-641.e7 (2019).

19. Schierding, W. et al. Common Variants Coregulate Expression of GBA and Modifier Genes to Delay Parkinson's Disease Onset. Mov. Disord. mds.28144 (2020). doi:10.1002/mds.28144

20. Liu, X., Li, Y. I. \& Pritchard, J. K. Trans Effects on Gene Expression Can Drive Omnigenic Inheritance. Cell 177, 1022-1034.e6 (2019).

21. Fadason, T., Schierding, W., Lumley, T. \& O’Sullivan, J. M. Chromatin interactions and expression quantitative trait loci reveal genetic drivers of multimorbidities. Nat. Commun. 9, 5198 (2018). 
22. Smemo, S. et al. Obesity-associated variants within FTO form long-range functional connections with IRX3. Nature 507, 371-375 (2014).

23. Nica, A. C. \& Dermitzakis, E. T. Expression quantitative trait loci: Present and future. Philosophical Transactions of the Royal Society B: Biological Sciences 368, (2013).

24. Barnat, M. et al. Huntington's disease alters human neurodevelopment. Science (80-. ). 793, eaax3338 (2020).

25. Fadason, T. et al. Reconstructing the blood metabolome and genotype using longrange chromatin interactions. Metab. Open 100035 (2020). doi:10.1016/j.metop.2020.100035

26. Aguet, F. et al. The GTEx Consortium atlas of genetic regulatory effects across human tissues. bioRxiv 787903 (2019). doi:10.1101/787903

27. Kong, S. \& Zhang, Y. Deciphering Hi-C: from 3D genome to function. Cell Biology and Toxicology 35, 15-32 (2019).

28. Schmitt, A. D. et al. A Compendium of Chromatin Contact Maps Reveals Spatially Active Regions in the Human Genome. Cell Rep. 17, 2042-2059 (2016).

29. Won, H. et al. Chromosome conformation elucidates regulatory relationships in developing human brain. Nature 538, (2016).

30. Dixon, J. R. et al. Chromatin architecture reorganization during stem cell differentiation. Nature 518, 331-336 (2015).

31. Dunham, I. et al. An integrated encyclopedia of DNA elements in the human genome. Nature 489, 57-74 (2012).

32. Walker, R. L. et al. Genetic Control of Expression and Splicing in Developing Human Brain Informs Disease Mechanisms. Cell 179, 750-771.e22 (2019).

33. Dayem Ullah, A. Z. et al. SNPnexus: assessing the functional relevance of genetic variation to facilitate the promise of precision medicine. Nucleic Acids Res. 46, 109- 
$113(2018)$

34. Karczewski, K. J. et al. The mutational constraint spectrum quantified from variation in 141,456 humans. Nature 581, 434-443 (2020).

35. Karczewski, K. J. et al. The mutational constraint spectrum quantified from variation in 141,456 humans. Genome Aggreg. Database Consort. 19, (2020).

36. Szklarczyk, D. et al. STRING v11: protein-protein association networks with increased coverage, supporting functional discovery in genome-wide experimental datasets. Nucleic Acids Res. 47, D607-D613 (2019).

37. Blondel, V. D., Guillaume, J. L., Lambiotte, R. \& Lefebvre, E. Fast unfolding of communities in large networks. J. Stat. Mech. Theory Exp. 2008, P10008 (2008).

38. Raudvere, U. et al. g:Profiler: a web server for functional enrichment analysis and conversions of gene lists (2019 update). Nucleic Acids Res. 47, W191-W198 (2019).

39. Makarious, M. B. et al. Multi-Modality Machine Learning Predicting Parkinson's Disease. bioRxiv 2, (2021).

40. Grenn, F. P. et al. The Parkinson's Disease Genome-Wide Association Study Locus Browser. Mov. Disord. 35, 2056-2067 (2020).

41. Westra, H.-J. et al. Systematic identification of trans eQTLs as putative drivers of known disease associations. Nat. Genet. 45, 1238-1243 (2013).

42. spicuglia, salvatore \& Vanhille, L. Chromatin signatures of active enhancers. Nucleus 3, 126-131 (2012).

43. Aguet, F. et al. The GTEx Consortium atlas of genetic regulatory effects across human tissues. Science (80-. ). 369, 1318-1330 (2020).

44. Karczewski, K. J. et al. Variation across 141,456 human exomes and genomes reveals the spectrum of loss-of-function intolerance across human protein-coding genes. bioRxiv 531210 (2019). doi:10.1101/531210 
45. Schwamborn, J. C. Is Parkinson's disease a neurodevelopmental disorder and will brain organoids help us to understand it? Stem Cells Dev. 27, 968-975 (2018).

46. Sulzer, D. Multiple hit hypotheses for dopamine neuron loss in Parkinson's disease. Trends in Neurosciences 30, 244-250 (2007).

47. Johnson, M. E., Stecher, B., Labrie, V., Brundin, L. \& Brundin, P. Triggers, Facilitators, and Aggravators: Redefining Parkinson's Disease Pathogenesis. Trends in Neurosciences 42, 4-13 (2019).

48. Lage, K. Protein-protein interactions and genetic diseases: The interactome. Biochimica et Biophysica Acta - Molecular Basis of Disease 1842, 1971-1980 (2014).

49. Yoshida, H. \& Goedert, M. Phosphorylation of microtubule-associated protein tau by AMPK-related kinases. J. Neurochem. 120, 165-176 (2012).

50. Fasano, D. et al. Alteration of endosomal trafficking is associated with early-onset parkinsonism caused by SYNJ1 mutations. Cell Death Dis. 9, 1-15 (2018).

51. Quadri, M. et al. Mutation in the SYNJ1 Gene Associated with Autosomal Recessive, Early-Onset Parkinsonism. Hum. Mutat. 34, 1208-1215 (2013).

52. Le Clainche, C. et al. A Hip1R-cortactin complex negatively regulates actin assembly associated with endocytosis. EMBO J. 26, 1199-1210 (2007).

53. Wilbur, J. D. et al. Actin Binding by Hip1 (Huntingtin-interacting Protein 1) and Hip1R (Hip1-related Protein) Is Regulated by Clathrin Light Chain. (2008). doi:10.1074/jbc.M802863200

54. Yang, Q. et al. Endocytic adaptor protein HIP1R controls intracellular trafficking of epidermal growth factor receptor in neuronal dendritic development. Front. Mol. Neurosci. 11, 1-17 (2018).

55. Shen, X. G. et al. Retinoic Acid-Induced Protein 14 (RAI14) Promotes mTORMediated Inflammation Under Inflammatory Stress and Chemical Hypoxia in a U87 
Glioblastoma Cell Line. Cell. Mol. Neurobiol. 39, 241-254 (2019).

56. Wolf, D. et al. Ankyrin repeat-containing N-Ank proteins shape cellular membranes. Nat. Cell Biol. 21, 1191-1205 (2019).

57. Orr, C. F., Rowe, D. B. \& Halliday, G. M. An inflammatory review of Parkinson's disease. Progress in Neurobiology 68, 325-340 (2002).

58. Clark, J. N., Whiting, A. \& McCaffery, P. Retinoic acid receptor-targeted drugs in neurodegenerative disease. Expert Opin. Drug Metab. Toxicol. 16, 1097-1108 (2020).

59. Bae, E. J. et al. LRRK2 kinase regulates $\alpha$-synuclein propagation via RAB35 phosphorylation. Nat. Commun. 9, (2018).

60. Beach, T. G. et al. Multi-organ distribution of phosphorylated alpha-synuclein histopathology in subjects with Lewy body disorders. Acta Neuropathol. 119, 689-702 (2010).

61. Mohammadi, S., Dolatshahi, M. \& Rahmani, F. Shedding light on thyroid hormone disorders and Parkinson disease pathology: mechanisms and risk factors. Journal of Endocrinological Investigation 1-13 (2020). doi:10.1007/s40618-020-01314-5

62. Mohammadi, S., Dolatshahi, M. \& Rahmani, F. Shedding light on thyroid hormone disorders and Parkinson disease pathology: mechanisms and risk factors. Journal of Endocrinological Investigation 1-13 (2020). doi:10.1007/s40618-020-01314-5

63. Chen, S. F., Yang, Y. C., Hsu, C. Y. \& Shen, Y. C. Risk of Parkinson's disease in patients with hypothyroidism: A nationwide population-based cohort study. Park. Relat. Disord. 74, 28-32 (2020).

64. Houser, M. C. \& Tansey, M. G. The gut-brain axis: Is intestinal inflammation a silent driver of Parkinson's disease pathogenesis? npj Parkinson's Disease 3, 1-9 (2017).

65. Suttrup, I. et al. Esophageal dysfunction in different stages of Parkinson's disease. Neurogastroenterol. Motil. 29, e12915 (2017). 
66. Wu, Z. Q. et al. Caspr Controls the Temporal Specification of Neural Progenitor Cells through Notch Signaling in the Developing Mouse Cerebral Cortex. Cereb. Cortex 27, 1369-1385 (2017).

67. Spratley, S. J. et al. Molecular Mechanisms of Disease Pathogenesis Differ in Krabbe Disease Variants. Traffic 17, 908-922 (2016).

68. Marshall, M. S. \& Bongarzone, E. R. Beyond Krabbe's disease: The potential contribution of galactosylceramidase deficiency to neuronal vulnerability in late-onset synucleinopathies. J. Neurosci. Res. 94, 1328-1332 (2016).

69. Sun, Y., Curle, A. J., Haider, A. M. \& Balmus, G. The role of DNA damage response in amyotrophic lateral sclerosis. Essays in Biochemistry 64, 847-861 (2020).

70. Wu, J., Lu, L. Y. \& Yu, X. The role of BRCA1 in DNA damage response. Protein and Cell 1, 117-123 (2010).

71. Liaw, H., Lee, D. \& Myung, K. DNA-PK-dependent RPA2 hyperphosphorylation facilitates DNA repair and suppresses sister chromatid exchange. PLoS One 6, 21424 (2011).

72. Wu, W. et al. Neuronal enhancers are hotspots for DNA single-strand break repair. Nature (2021). doi:10.1038/s41586-021-03468-5

73. Mei, Y. et al. Identification of BECN1 and ATG14 Coiled-Coil Interface Residues That Are Important for Starvation-Induced Autophagy. Biochemistry 55, 4239-4253 (2016).

74. Tan, E. K. et al. Parkinson disease and the immune system - associations, mechanisms and therapeutics. Nature Reviews Neurology 16, 303-318 (2020).

75. Yu, E. et al. Fine mapping of the HLA locus in Parkinson's disease in Europeans. medRxiv 2020.10.29.20217059 (2020). doi:10.1101/2020.10.29.20217059

76. Koussounadis, A., Langdon, S. P., Um, I. H., Harrison, D. J. \& Smith, V. A. 
bioRxiv preprint doi: https://doi.org/10.1101/2021.04.08.439080; this version posted April 9, 2021. The copyright holder for this preprint (which was not certified by peer review) is the author/funder, who has granted bioRxiv a license to display the preprint in perpetuity. It is made available under aCC-BY-NC-ND 4.0 International license.

Relationship between differentially expressed mRNA and mRNA-protein correlations

in a xenograft model system. Sci. Rep. 5, 1-9 (2015). 


\section{Figures}

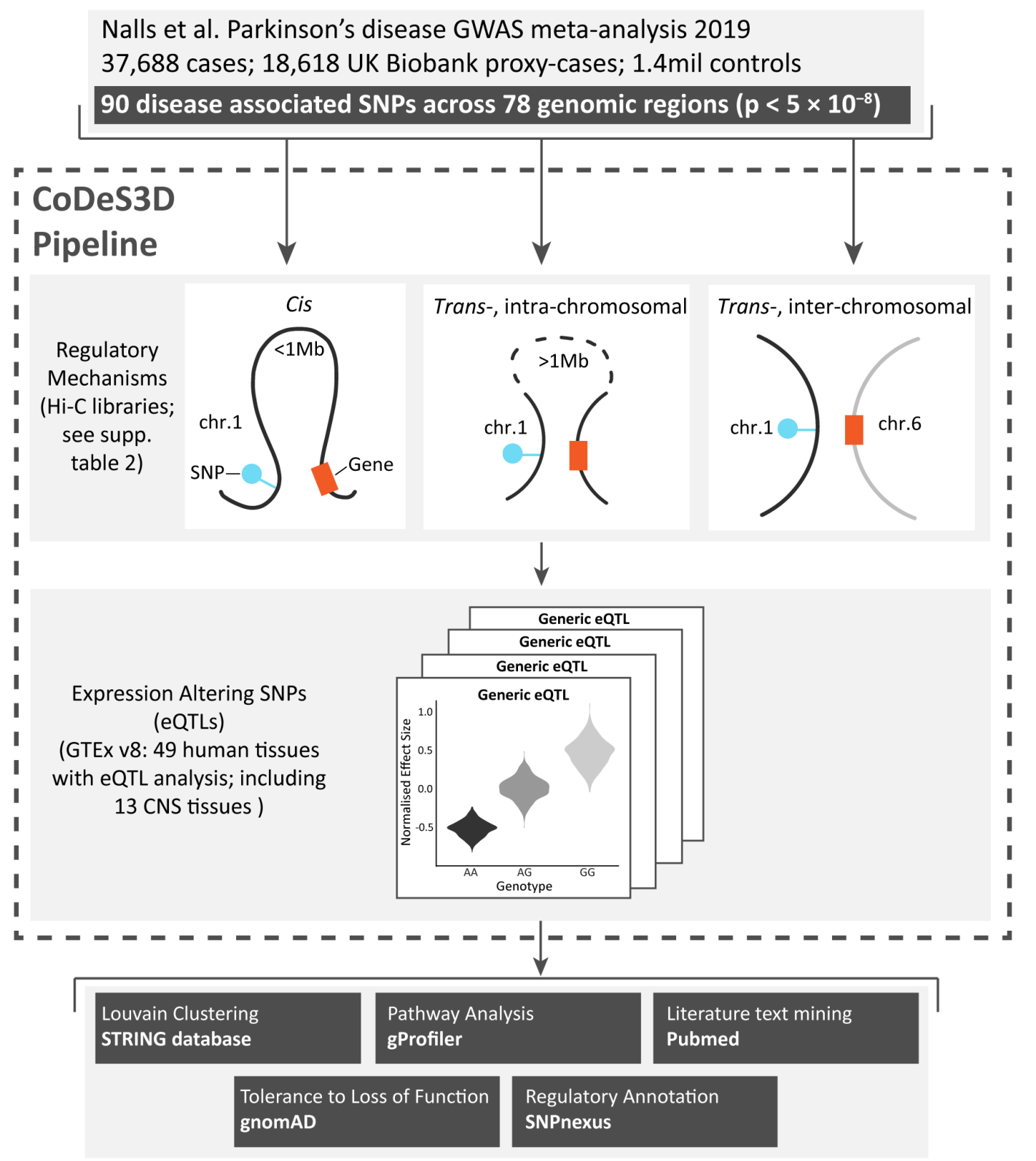

Figure 1: Methods workflow. 90 PD-SNPs were obtained from Nalls et al ${ }^{5}$. Spatial interactions between the 90 PD-SNPs and genes were identified from Hi-C libraries (Supplementary table 2). The resulting spatial SNP-gene pairs were then used to query GTEx v8 to identify significant eQTLs. The resulting SNP-gene pairs were then analysed for functional relevance using multiple tools and databases (methods). Figure adapted from Schierding et al. ${ }^{19}$ 
bioRxiv preprint doi: https://doi.org/10.1101/2021.04 08.439080; this version posted April 9, 2021. The copyright holder for this preprint (which was not certified by peer review) is the author/funder, who has granted bioRxiv a license to display the preprint in perpetuity. It is made available under aCC-BY-NC-ND 4.0 International license.

\begin{tabular}{|l|}
\hline eQTL distance \\
All eQTLs \\
Cis \\
Trans- Intrachromosomal \\
Trans- Interchromosomal \\
\hline
\end{tabular}

All eQTLsregulation

GTEx Brain Tissues
Amygdala
Anterior cingulate cortex (BA24)
Caudate (basal ganglia)
Cerebellar Hemisphere
Cerebellum
Cortex
Frontal Cortex (BA9)
Hippocampus
Hypothalamus
Nucleus accumbens (basal ganglia)
Putamen (basal ganglia)
Spinal cord (cervical c-1)
Subatantia nigra

Cis- regulation

Trans-

intrachromosomalregulation

Trans-

interchromosomalregulation
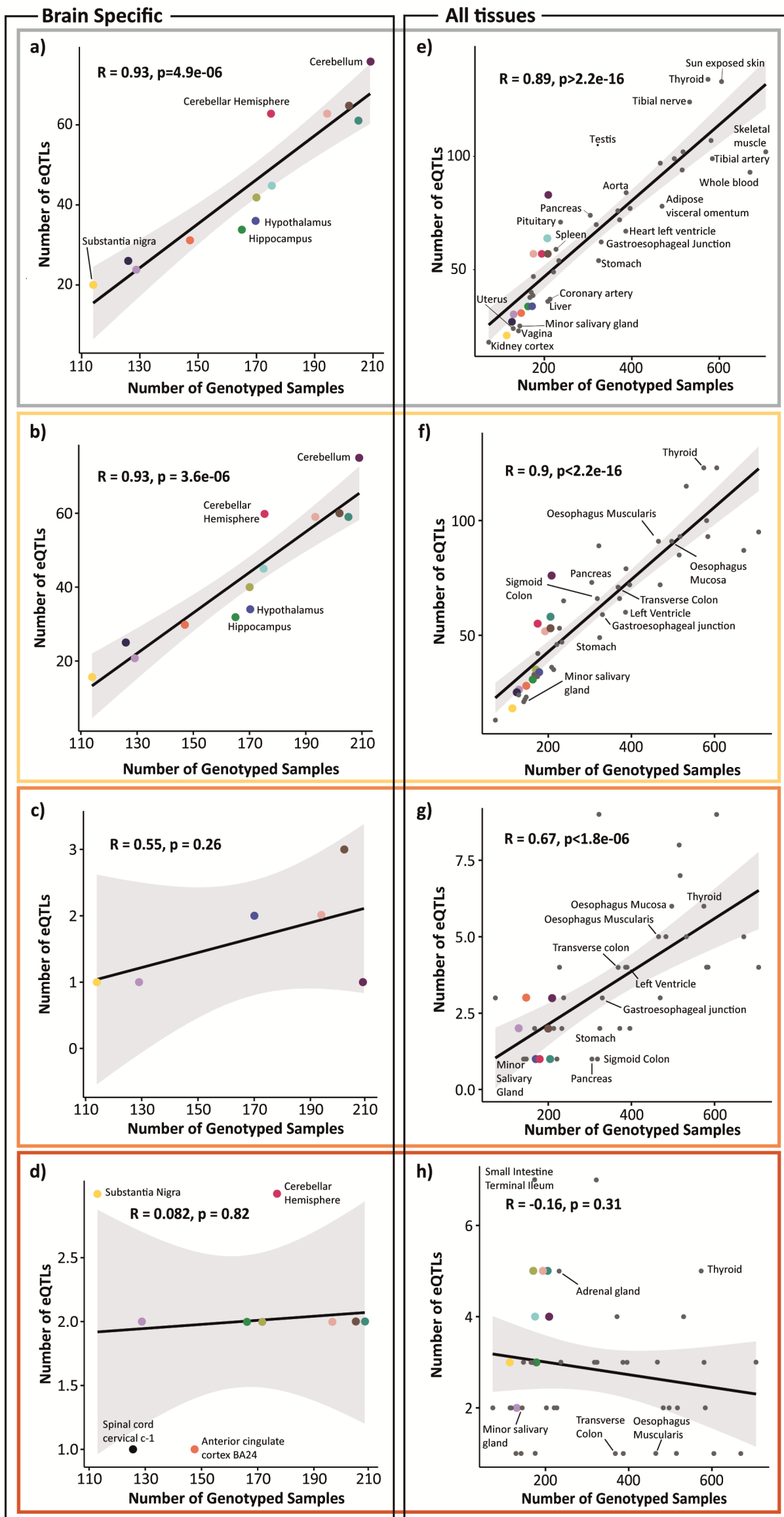


\section{Figure 2: Correlation between genotype samples per tissue and number of}

eQTLs present in the tissue (a) Correlation between the number of genotyped samples per tissue (in GTEx) and the number of eQTLs (including cis, trans-intrachromosomal and trans-interchromosomal) per tissue, in 13 brain-specific tissues (b) Correlation between the number of genotyped samples per tissue (in GTEx) and the number of cis-eQTLs per tissue, in 13 brain-specific tissues (c) Correlation between the number of genotyped samples per tissue (in GTEx) and the number of trans-intrachromosomal-eQTLs per tissue, in 13 brainspecific tissues (d) Correlation between the number of genotyped samples per tissue (in GTEx) and the number of trans-interchromosomal-eQTLs per tissue, in 13 brain-specific tissues (e) Correlation between the number of genotyped samples per tissue (in GTEx) and the number of eQTLs (including cis, trans-intrachromosomal and trans-interchromosomal) per tissue, in all 49 tissues (f) Correlation between the number of genotyped samples per tissue (in GTEx) and the number of cis-eQTLs per tissue, in all 49 tissues (g) Correlation between the number of genotyped samples per tissue (in GTEx) and the number of transintrachromosomal-eQTLs per tissue, in all 49 tissues (h) Correlation between the number of genotyped samples per tissue (in GTEx) and the number of trans-interchromosomal-eQTLs per tissue, in all 49 tissues. The tissues that fall furthest from the confidence interval are annotated. The grey dots show the correlation for all GTEx tissues. The 13 brain tissues (from GTEx) are indicated by the coloured dots, as shown in the legend. For information on all tissues outside of the 95\% CI see Supplementary table 12 . 


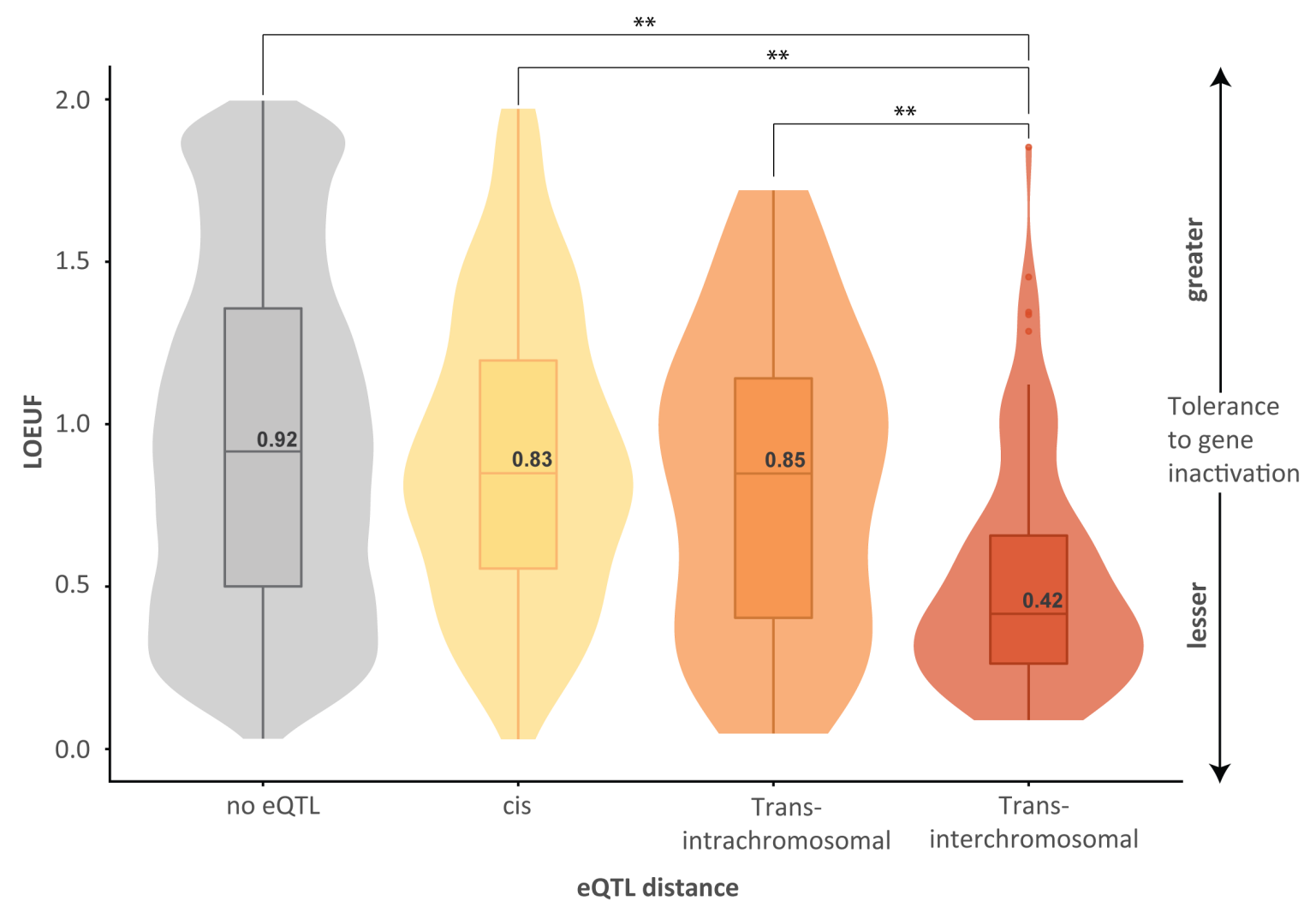

\section{Figure 3: Genes subjected to trans-regulation by PD-SNPs are enriched for Loss-}

of-Function Intolerance. Genes that are loss of function intolerant, as measured by a continuous LOEUF score, are enriched in trans-regulatory interactions involving PD-SNPs.

The LOEUF score is a continuous value that indicates the tolerance of a given gene to inactivation. Low LOEUF scores indicate stronger selection against loss-of-function variation. The distribution is shown as a violin plot with the median (LOEUF) values for each eQTL group (black text). The groups were compared using a Kruskal-Wallis test $(* *=P$ value $<0.01)$; the absence of a significance value indicates the LOEUF values of the two groups were not significantly different. No eQTL = all genes in gnomAD with an assigned pLI or LOEUF for which an eQTL was not identified in this study $(\sim 18,500$ genes $)$. Not all genes had LOEUF scores (Supplementary figure 2; Supplementary table 7) 


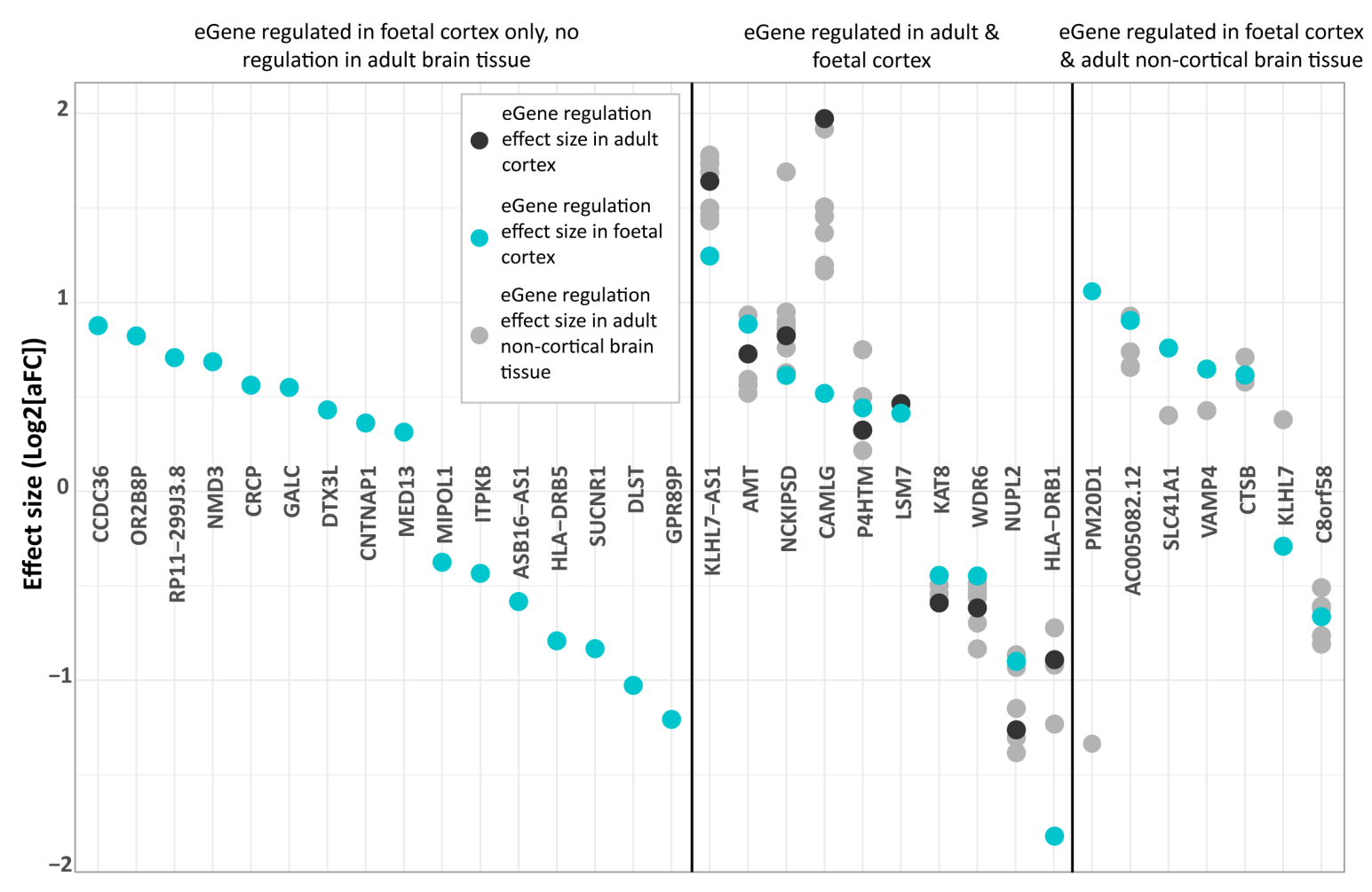

Figure 4: Gene regulation in the foetal cortex compared to the adult cortex. The

leftmost section shows genes that are regulated only in the foetal cortex, with no eQTLs seen in any of the 13 adult brain tissues. The middle section shows genes that are regulated in both the foetal and adult cortex. The black dots show the regulation effect size of the gene in the adult cortex, and the grey dots show the regulation effect size of the gene across the different brain tissues (where an eQTL is seen). The rightmost section shows genes that are regulated in both the foetal cortex and adult non-cortical brain tissue. 
bioRxiv preprint doi: https://doi.org/10.1101/2021.04 08 439080; this version posted April 9, 2021. The copyright holder for this preprint (which was not certified by peer review) is the author/funder, who has granted bioRxiv a license to display the preprint in perpetuity. It is made available under aCC-BY-NC-ND 4.0 International license.
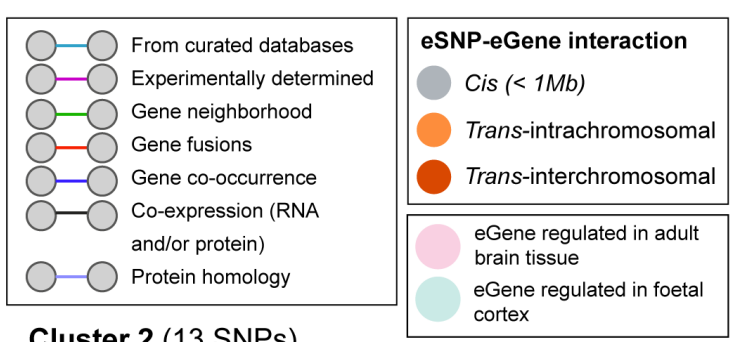

Cluster 2 (13 SNPs)

Notch signalling pathway

WNT Signalosome

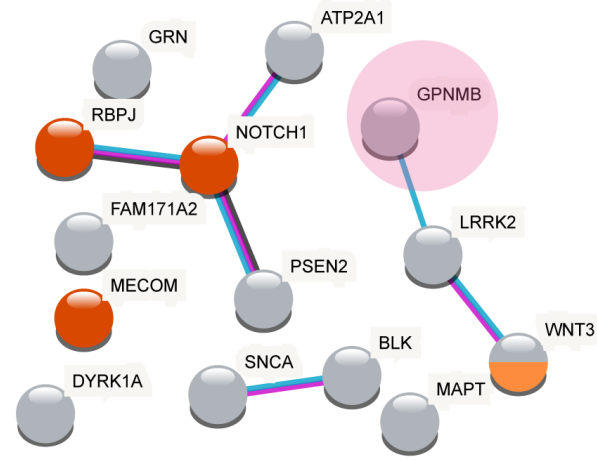

Cluster 3 (6 SNPs)

Microtubule polymerisation

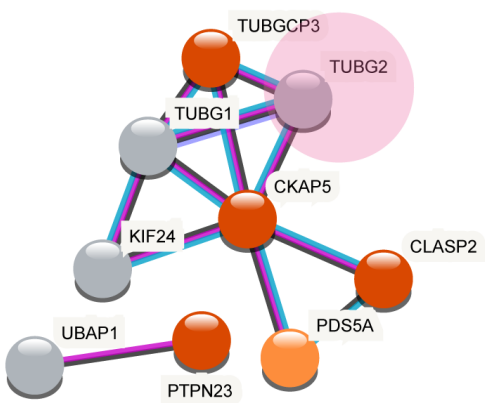

WNT3

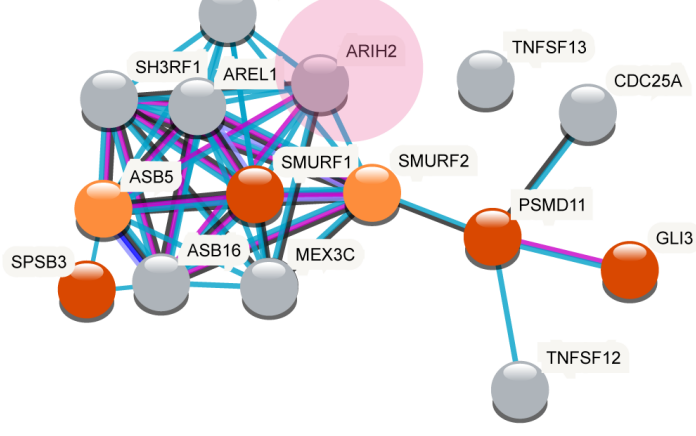

Cluster 4 (13 SNPs)

Protein modification process

Vesicle mediated transport
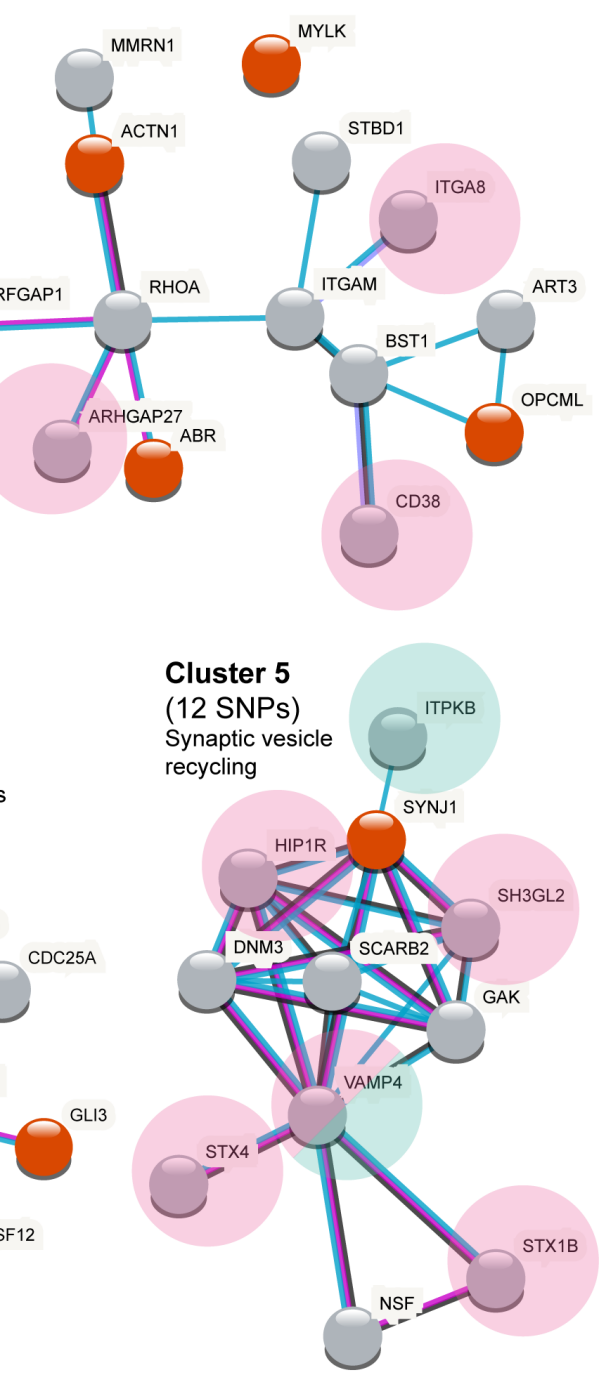
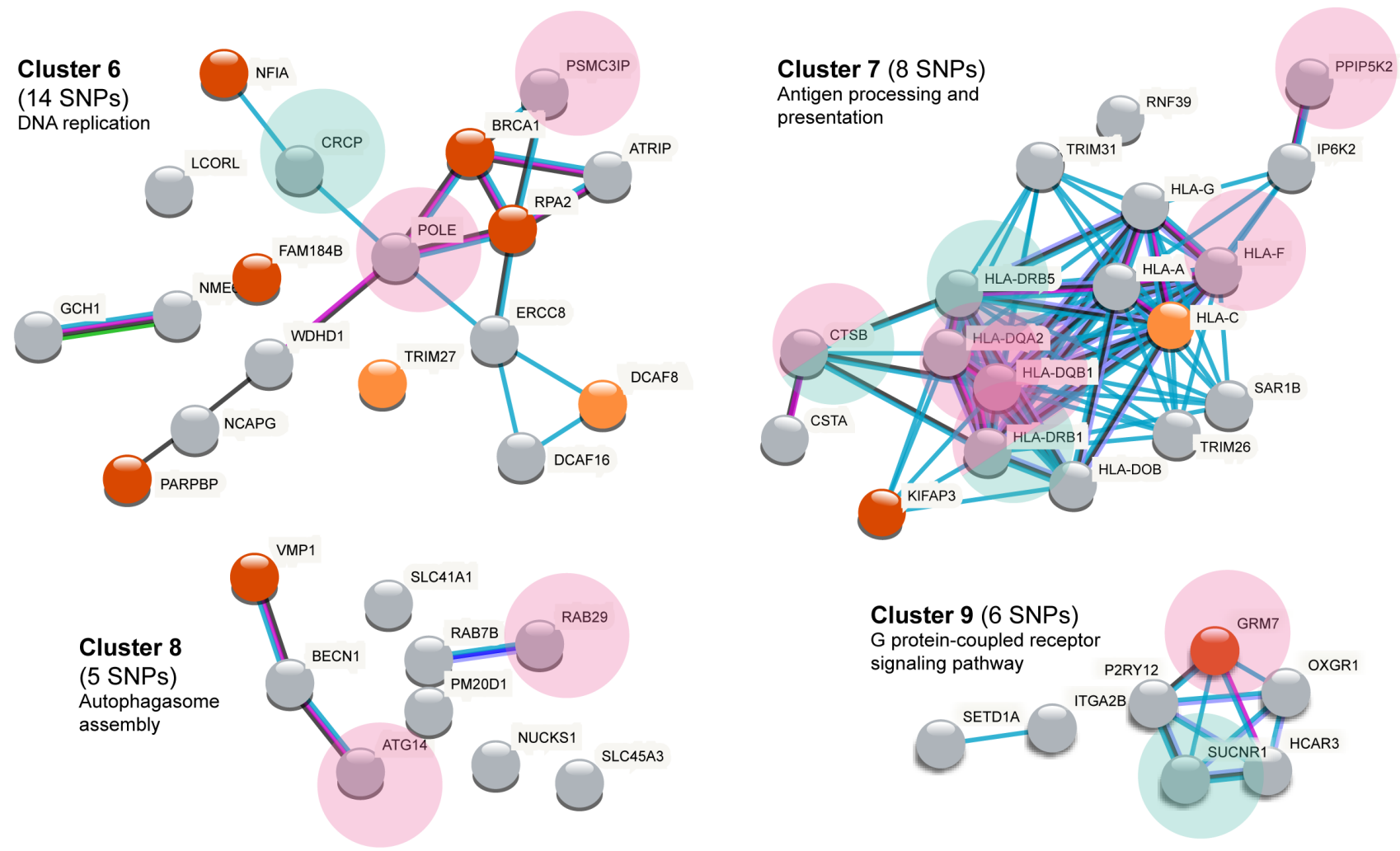


\section{Figure 5: Louvain Clustering analysis highlights nine significant clusters,}

indicative of biological connectivity. The grey and orange shading of the nodes is indicative of whether the gene is subject to regulation via cis- or trans- mechanisms. The pink and turquoise shaded circles indicate genes that are regulated in adult brain tissue and foetal cortex respectively. STRING PPI confidence level = high (0.700); text-mining connections removed. The clusters were also analysed in STRING with an increased stringency (including only experimentally determined and curated database connections, confidence level: 0.700 ); however, this led to very few changes, with cluster 6 the only cluster to lose any connectivity within the cluster (WDHD1, NCAPG and PARPBP no longer connect). Experimentally determined: imported from experimental repositories; Gene neighbourhood: similar genomic context in different species suggest a similar function of the proteins; Gene fusions: fused proteins are recognised by orthology of the fused parts to other, non-fused proteins; Gene cooccurrence: indicates the presence of a specific gene pair is in agreement in all species - must be expressed together; Co-expression: predicted association between genes/proteins based on RNA and/or protein expression. 


\section{Supplementary Figures}

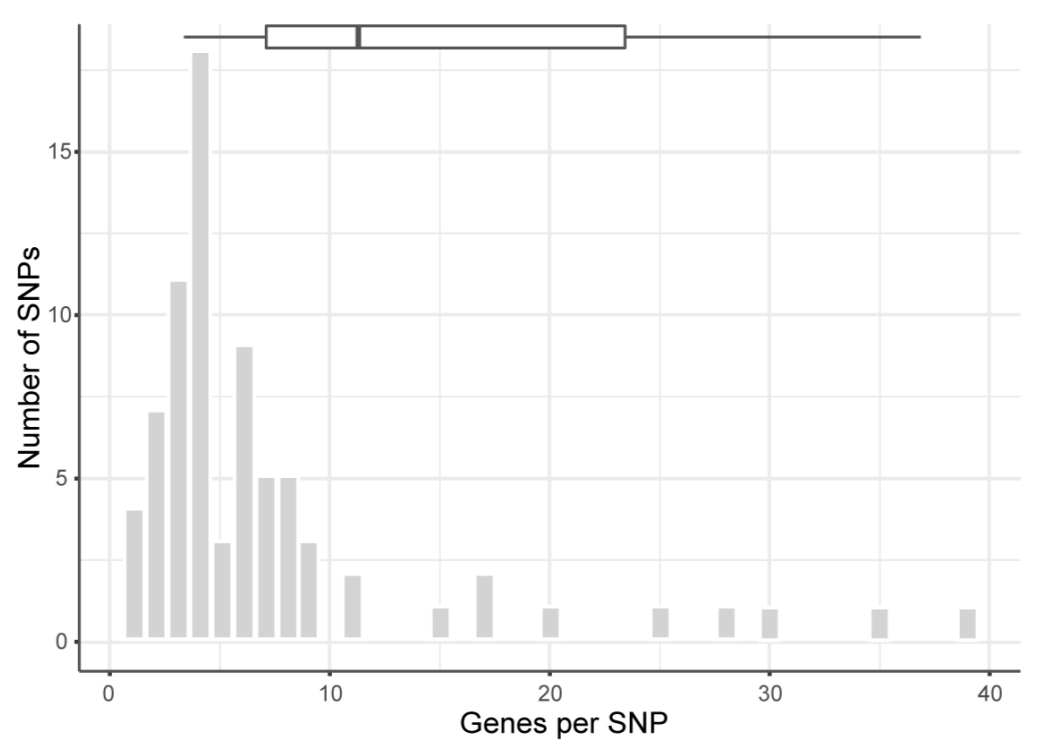

Supplementary figure 1: Number of genes regulated per SNP. Each of the 76 SNPs

has an eQTL with between 1 and 39 genes. The boxplot represents the median and interquartile range.

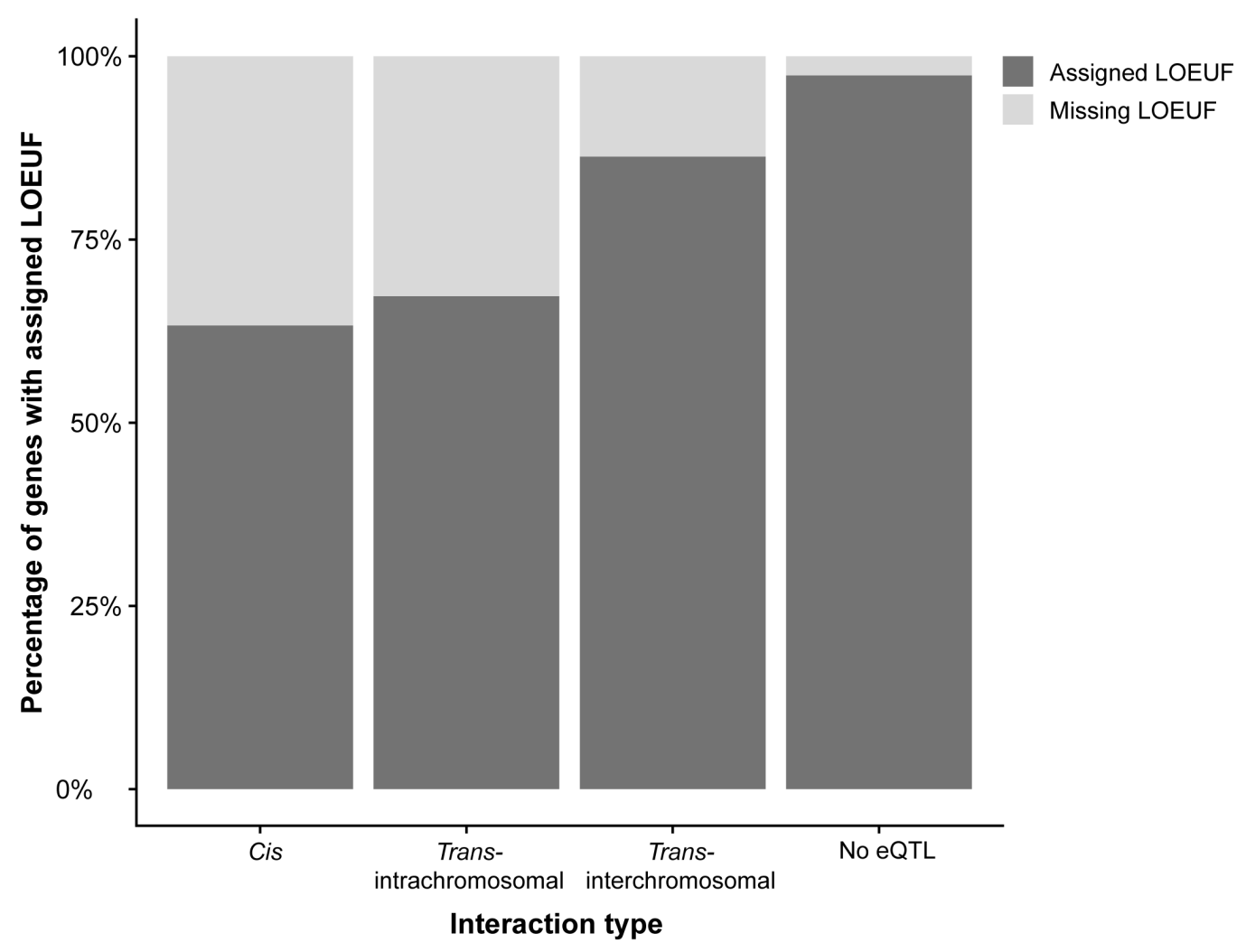




\section{Supplementary figure 2: The proportion of genes that are loss of function}

intolerant increases as the eQTL distance increases. Not all genes have an assigned

LOEUF score. The plot shows the percentage of genes that have no assigned score for each of the eQTL categories.

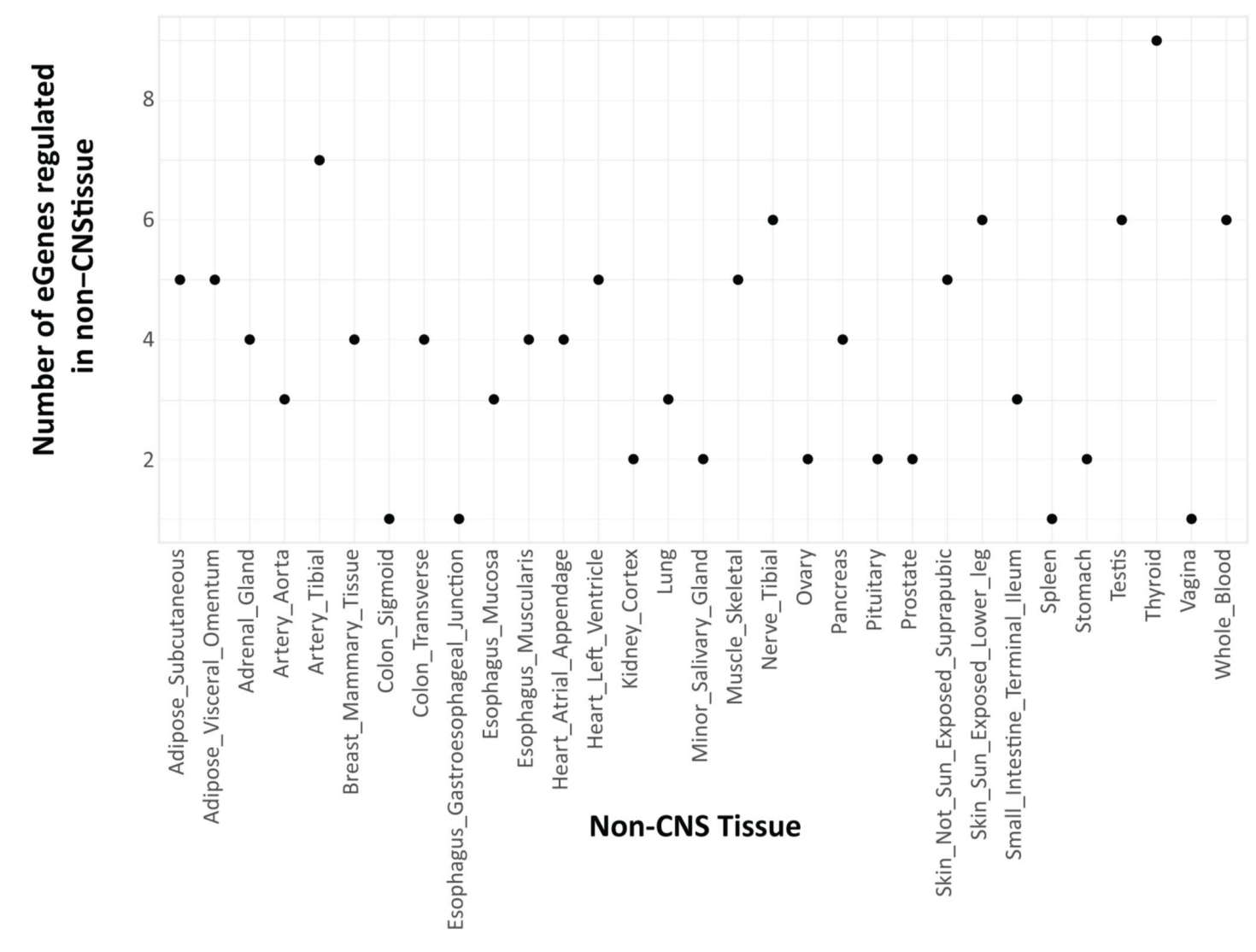

Supplementary figure 3: Genes subject to regulation in non-CNS tissues only. Our analysis identified a subset of 21 PD GWAS SNPs that regulate genes only within non-CNS tissues (i.e. these SNPs had no regulatory connections within brain tissues). 
bioRxiv preprint doi: https://doi.org/10.1101/2021.04 08.439080; this version posted April 9, 2021. The copyright holder for this preprint (which was not certified by peer review) is the author/funder, who has granted bioRxiv a license to display the preprint in perpetuity. It is made available under aCC-BY-NC-ND 4.0 International license.

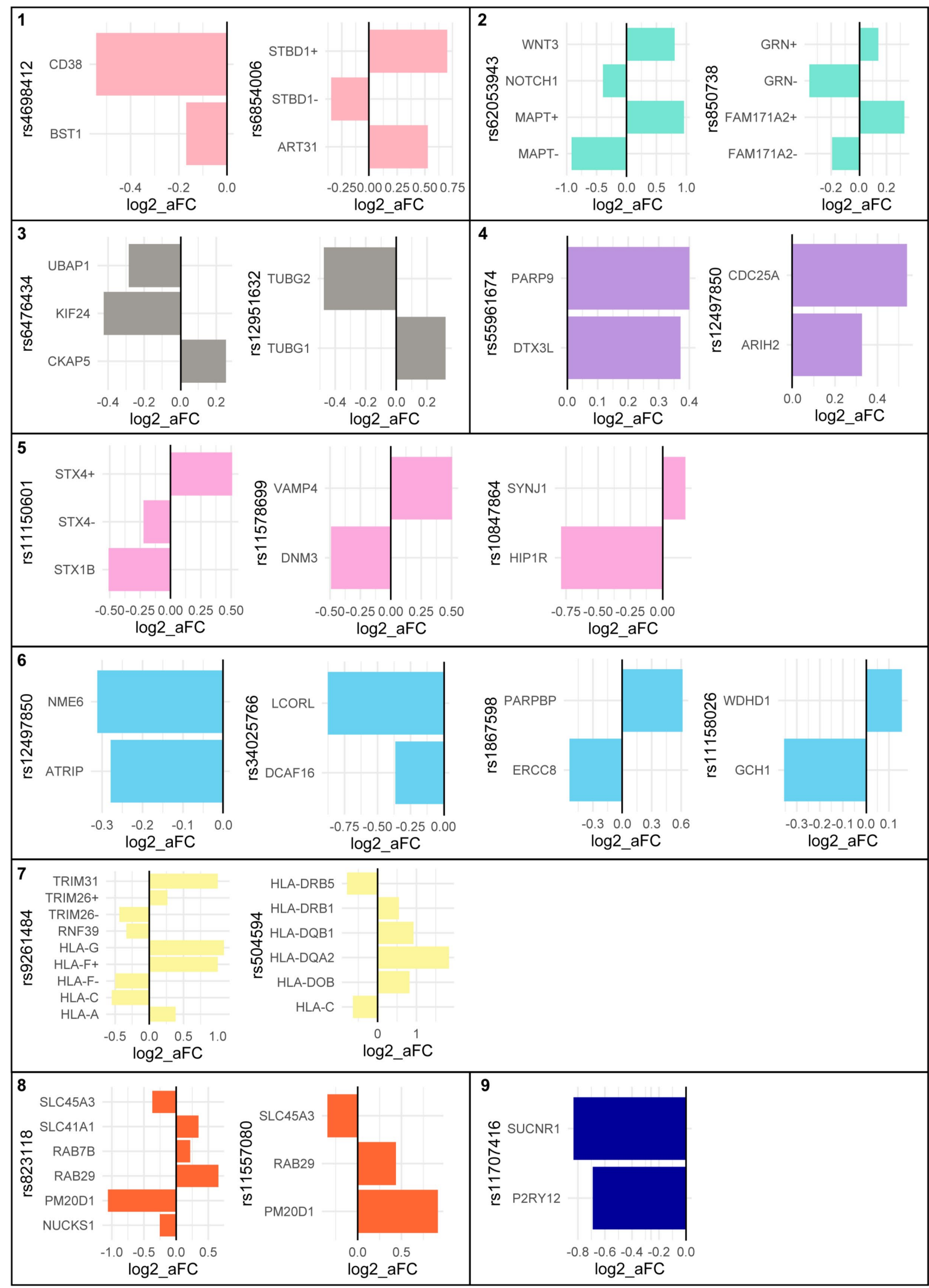




\section{Supplementary figure 4: SNPs co-regulate multiple genes within individual}

clusters. For each of the seven clusters there is at least one SNP that co-regulates more than one of the genes within that cluster. The SNPs do not co-regulate the genes in the same direction in most instances. In some cases where the SNP regulates the expression of one gene in multiple tissues, the regulation may be positive in some tissues, but negative in others. For example expression of STBD1 is down-regulated in 7 tissues but up-regulated in the testis and lung.

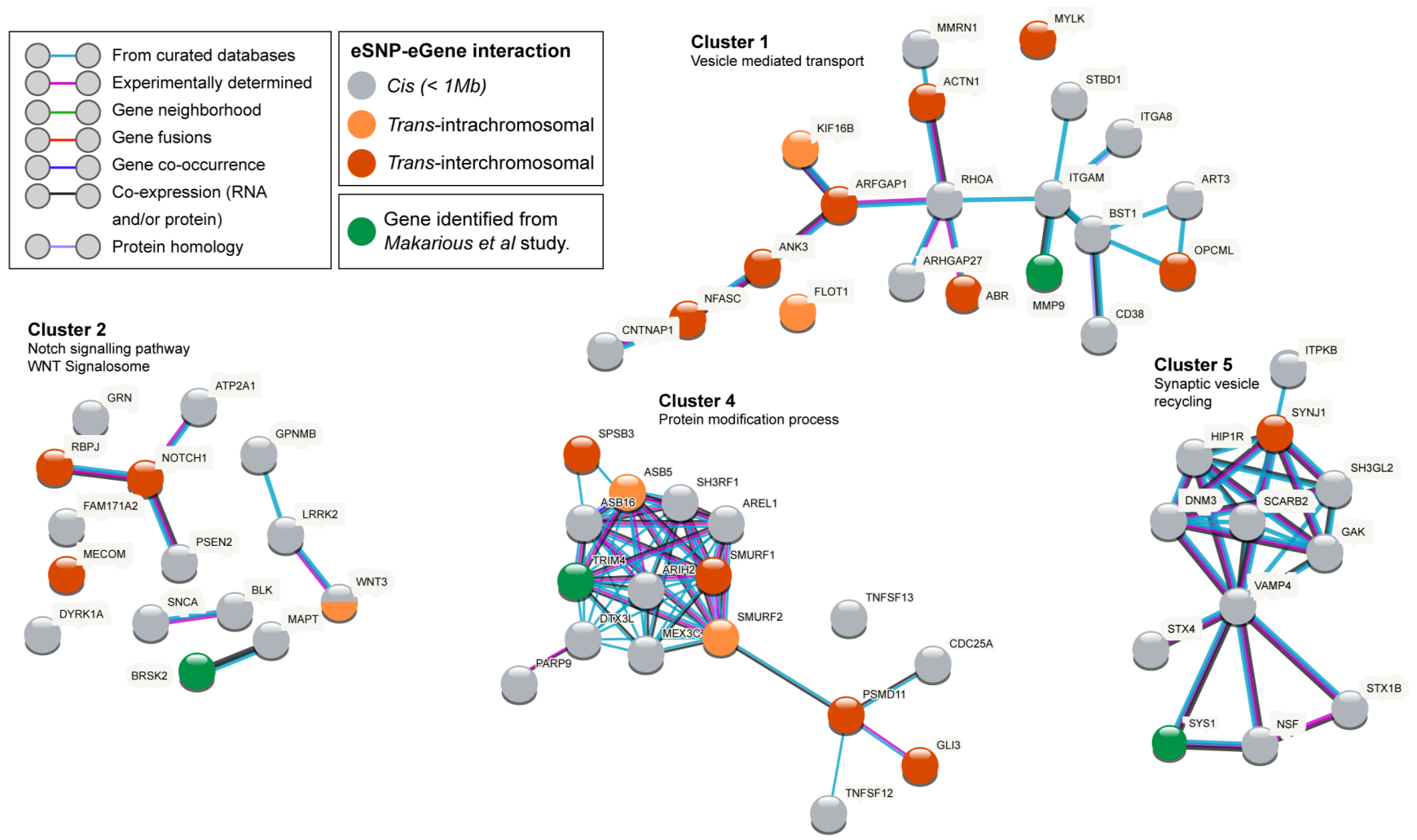

\section{Supplementary figure 5: Genes and variants identified by Makarious et al}

connect into clusters 1, 2, 4 and 5. Makarious et al identified a set of genes and variants

that affect the polygenic risk score for diagnosis of PD. A subset of these genes connect into the clusters identified through our analysis, showing a convergence between the two datasets, and further confirming the importance of the enriched pathways to PD biology. Adapted from main figure 5. The green shading of the nodes indicates genes that are identified from the Makarious et al dataset. 